\title{
Editorial
}

\section{Progesterone: the key to success?}

\section{Arianna Riva ${ }^{1, *}$, Giovanni Buzzaccarini ${ }^{1}$, Amerigo Vitagliano ${ }^{1}$, Antonio Simone Laganà ${ }^{2}$, Gaspare Cucinella ${ }^{3}$, Giuseppe Gullo ${ }^{3}$}

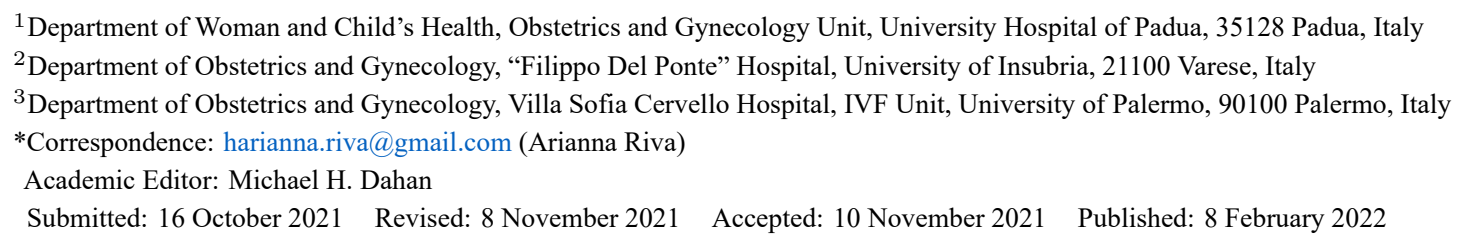

Infertility is a global health issue, affecting over $10 \%$ of women worldwide [1,2]. Infertility has psychological, medical and economic implications, resulting in trauma and stress in couples requiring IVF [3,4]. Many causes of infertility, in both women and men, have to be taken into account, ranging from daily habits, environmental factors, infections, hormonal, genetic and anatomical pathologies $[5,6]$. Assisted reproductive technologies (ARTs) are commonly used globally with several forms of interventions, which are ever more advanced and attempt to address the challenges linked to the broad spectrum of infertility in humans. Treatments include in vitro handling of human oocytes, spermatozoa, embryos and donors' gametes or embryos [7-11]. For a pregnancy to occur, endocrinological changes during the luteal phase need to be synchronised with endometrial maturation. The period when the endometrium can receive the embryo is called the window of implantation. Its individualisation is crucial to improving pregnancy rates.

Endogenous luteal phase support is impaired in IVF cycles and exogenous supplementation is mandatory [1214]. After the ovulation trigger with hCG, a supraphysiological and rapid rise in progesterone occurs four days after oocyte retrieval and falls on the sixth day after oocyte pickup, just before the opening of the implantation window. After the GnRH agonist trigger, progesterone growth reaches an almost physiological concentration, but a complete and irreversible luteolysis takes place within five days [15].

Studies conducted on the luteal phase support after GnRH antagonist trigger help us to comprehend the central role of serum progesterone concentration in the luteal phase and its importance on the implantation window and the reproductive outcome: a very low level of mid-luteal serum progesterone (OPU +7 days) [16] is related to a very high percentage of pregnancy losses $(79 \%)$. In the following studies, the authors attempt to prove that, by adding low doses of hCG, corpora lutea are recovered $[17,18]$. An increase in serum progesterone improves clinical pregnancy rates (CPR) and reduces pregnancy loss rates.

In the case, where luteal serum progesterone could predict pregnancy, as Thomsen et al. [19] reported in a prospective study of $602 \mathrm{IVF} / \mathrm{ICSI}$ cycles: $40 \%$ of patients had a reduced probability of a positive reproductive outcome below and above a mid-luteal serum progesterone of 150-250 nmol/L (47-79 ng/mL).

Similarly, low serum progesterone levels $(<8.8$ $\mathrm{ng} / \mathrm{mL}$ ) on the day of embryo transfer in the frozen artificial cycles have been related to a negative impact on pregnancy outcomes when using micronized vaginal progesterone, in both the donated egg and the subject's oocytes [20,21]. In a retrospective study, Kofinas et al. [22] find comparable results, supplementing the luteal phase utilising intramuscular progesterone: progesterone level on the ET day below 20 $\mathrm{ng} / \mathrm{dL}$ may cause a significantly lower ongoing pregnancy rate (OPR) and live birth rate (LBR). There is also a significant decrease in OPR and LBR when progesterone crosses the $40 \mathrm{ng} / \mathrm{mL}$.

The role of luteal progesterone during the implantation period is important, but we still do not know the exact mechanism of its action. To better understand the concept, two different studies are relevant. Young et al. [23] have focused their attention on secretory endometrial histology and endometrial gene expression in patients who received different doses of intramuscular progesterone $(2.5 ; 5 ; 10$ or $40 \mathrm{mg}$ ) over a period of 10 days, compared to ovulatory control patients. Serum progesterone is also measured at its peak ( $2-3 \mathrm{~h}$ after injection) and trough $(1-2 \mathrm{~h}$ before injection) on two separate occasions between 3 and 10 days of progesterone treatment: only patients with higher serum progesterone level (belonging to the $40 \mathrm{mg}$ group) express an endometrial dating, comparable to natural cycle control women, while adequate histology is seen in all groups, except for those with lower progesterone $(2.5 \mathrm{mg})$. These findings support the hypothesis that serum progesterone is the best marker of endometrial receptivity. Whether this receptivity is gained due to a direct effect on endometrium or systemically, is the subject of a recent study [24]: 79 patients underwent endometrial preparation with valerate oestradiol from day 1 or 2 and vaginal progesterone when endometrium reaches a thickness of $6.5 \mathrm{~mm}$. After five days of progesterone supplementation, a blood sample and an endometrial biopsy are collected. Measurements include serum progesterone, ERA test for the evaluation of endometrial receptivity and endometrial progesterone, with 17 hy- 
droxyprogesterone, oestrone and oestradiol. Surprisingly, no relationship between serum progesterone and endometrial receptivity (ERA test) is observed, while endometrial progesterone and 17 hydroxyprogesterone are related to the ERA test. Serum progesterone levels seem to be crucial in supporting and helping gestation, through immunological adaptation systems, while endometrial progesterone ensures its receptivity. Different ways of administration establish very different concentrations of serum progesterone $[25,26]$, due to a reaction to pharmacokinetic effects, such as absorption, distribution, metabolism and excretion. Vaginal administration of progesterone results in higher concentrations of the drug in the uterine tissue, through some form of a direct transport mechanism, named "first uterine pass effect" [27]. The greatest tissue concentration corresponds to a lower serum circulating progesterone level, while intramuscular administration leads to optimal plasma concentrations. It is noteworthy that in the study by Young et al. [23], progesterone is administered intramuscularly, while in the study by Labarta et al. [24], it is only administered vaginally: this could highlight an important role for the mode of administration, not just for serum progesterone concentration. It opens the possibility that two administration ways (e.g., vaginal and s.c. or i.m) are better than one (e.g., vaginal). Moreover, we do not know whether progesterone administered systemically (e.g., intramuscular or subcutaneous) implies a correlation between endometrial progesterone, serum progesterone and endometrial genes, nor if the serum progesterone cut-off level might be different depending on the mode of administration.

The detection of all these mechanisms and the identification of the different ranges and cut-offs of serum progesterone might have a big impact on our daily practice by simply performing a blood test. Labarta et al. [28] and Cédrin-Durnerin [29] have proved a normalisation of OPR and LBR, giving additional progesterone in FRET cycles. The implications of these results will encourage us to consider how tailored treatments are the best option for a subgroup of patients.

\section{Author contributions}

$\mathrm{AR}$ - responsible for the concept and draft of the article; GB, AV-participate in the writing and editing of the article; ASL, GC and GG-contribution in manuscript revision.

\section{Ethics approval and consent to participate}

Not applicable.

\section{Acknowledgment}

Thanks to all the peer reviewers for their opinions and suggestions.

\section{Funding}

This research received no external funding.

\section{Conflict of interest}

The authors declare no conflict of interest. AV, ASL are serving as one of the Editorial Board members. GB is serving as one of the Guest editors. We declare that they had no involvement in the peer review of this article and has no access to information regarding its peer review. Full responsibility for the editorial process for this article was delegated to MD.

\section{References}

[1] WHO-ICMART revised glossary. 2021. Available at: https://www.who.int/reproductivehealth/publications/infertilit y/art_terminology2/en/ (Accessed: 16 October 2021).

[2] Mascarenhas MN, Flaxman SR, Boerma T, Vanderpoel S, Stevens GA. National, regional, and global trends in infertility prevalence since 1990: a systematic analysis of 277 health surveys. PLoS Medicine. 2012; 9: e1001356.

[3] Gullo G, Cucinella G, Perino A, Gullo D, Segreto D, Laganà AS, et al. The Gender Gap in the Diagnostic-Therapeutic Journey of the Infertile Couple. International Journal of Public Health. 2021; 18: 6184.

[4] La Rosa VL, Valenti G, Sapia F, Gullo G, Maria A, Rapisarda C. Psychological impact of gynecological diseases: the importance of a multidisciplinary approach. Italian Journal of Gynaecology and Obstetrics. 2018; 30: 23-26.

[5] Menichini D, Forte G, Orrù B, Gullo G, Unfer V, Facchinetti $\mathrm{F}$. The role of vitamin $\mathrm{D}$ in metabolic and reproductive disturbances of polycystic ovary syndrome: A narrative mini-review. International Journal for Vitamin and Nutrition Research. 2020. (in press)

[6] National Collaborating Centre for Women's and Children's Health (UK). Fertility: Assessment and Treatment for People with Fertility Problems. London: Royal College of Obstetricians \& Gynaecologists. 2013.

[7] Gullo G, Petousis S, Papatheodorou A, Panagiotidis Y, Margioula-Siarkou C, Prapas N, et al. Closed vs. Open Oocyte Vitrification Methods Are Equally Effective for Blastocyst Embryo Transfers: Prospective Study from a Sibling Oocyte Donation Program. Gynecologic and Obstetric Investigation. 2020; 85: 206-212.

[8] Pedrosa ML, Furtado MH, Ferreira MCF, Carneiro MM. Sperm selection in IVF: the long and winding road from bench to bedside. JBRA Assisted Reproduction. 2020; 24: 332-339.

[9] Sciorio R, Tramontano L, Catt J. Preimplantation genetic diagnosis (PGD) and genetic testing for aneuploidy (PGT-a): status and future challenges. Gynecological Endocrinology. 2020; 36: 6-11.

[10] Letterie G, Mac Donald A. Artificial intelligence in in vitro fertilization: a computer decision support system for day-to-day management of ovarian stimulation during in vitro fertilization. Fertility and Sterility. 2020; 114: 1026-1031.

[11] Goudakou M, Kalogeraki A, Matalliotakis I, Panagiotidis Y, Gullo G, Prapas Y. Cryptic sperm defects may be the cause for total fertilization failure in oocyte donor cycles. Reproductive Biomedicine Online. 2012; 24: 148-152.

[12] Fauser BC, Devroey P. Reproductive biology and IVF: ovarian stimulation and luteal phase consequences. Trends in Endocrinology and Metabolism. 2003; 14: 236-242.

[13] Fertility: Evidence Update March 2015. London: National Institute for Health and Care Excellence (UK). 2015. 
[14] Bosch E, Broer S, Griesinger G, Grynberg M, Humaidan P, Kolibianakis E, et al. ESHRE guideline: ovarian stimulation for IVF/ICSI $\uparrow$. Human reproduction open. 2020; 2020: hoaa009.

[15] Fatemi HM, Popovic-Todorovic B. Implantation in assisted reproduction: a look at endometrial receptivity. Reproductive Biomedicine Online. 2013; 27: 530-538.

[16] Humaidan P, Bredkjaer HE, Bungum L, Bungum M, Grøndahl ML, Westergaard L, et al. GnRH agonist (buserelin) or hCG for ovulation induction in GnRH antagonist IVF/ICSI cycles: a prospective randomized study. Human Reproduction. 2005; 20: $1213-1220$.

[17] Humaidan P, Ejdrup Bredkjær H, Westergaard LG, Yding Andersen C. 1,500 IU human chorionic gonadotropin administered at oocyte retrieval rescues the luteal phase when gonadotropinreleasing hormone agonist is used for ovulation induction: a prospective, randomized, controlled study. Fertility and Sterility. 2010; 93: 847-854.

[18] Humaidan P, Polyzos NP, Alsbjerg B, Erb K, Mikkelsen AL, Elbaek HO, et al. GnRHa trigger and individualized luteal phase hCG support according to ovarian response to stimulation: two prospective randomized controlled multi-centre studies in IVF patients. Human Reproduction. 2013; 28: 2511-2521.

[19] Thomsen LH, Kesmodel US, Erb K, Bungum L, Pedersen D, Hauge $\mathrm{B}$, et al. The impact of luteal serum progesterone levels on live birth rates - a prospective study of $602 \mathrm{IVF} / \mathrm{ICSI}$ cycles. Human Reproduction. 2018; 33: 1506-1516.

[20] Labarta E, Mariani G, Holtmann N, Celada P, Remohí J, Bosch E. Low serum progesterone on the day of embryo transfer is associated with a diminished ongoing pregnancy rate in oocyte donation cycles after artificial endometrial preparation: a prospective study. Human Reproduction. 2017; 32: 2437-2442.

[21] Labarta E, Mariani G, Paolelli S, Rodriguez-Varela C, Vidal C, Giles J, et al. Impact of low serum progesterone levels on the day of embryo transfer on pregnancy outcome: a prospective cohort study in artificial cycles with vaginal progesterone. Human Reproduction. 2020; 36: 683-692.

[22] Kofinas JD, Blakemore J, McCulloh DH, Grifo J. Serum progesterone levels greater than $20 \mathrm{ng} / \mathrm{dl}$ on day of embryo transfer are associated with lower live birth and higher pregnancy loss rates. Journal of Assisted Reproduction and Genetics. 2015; 32: 1395-1399.

[23] Young SL, Savaris RF, Lessey BA, Sharkey AM, Balthazar U, Zaino RJ, et al. Effect of randomized serum progesterone concentration on secretory endometrial histologic development and gene expression. Human Reproduction. 2017; 32: 1903-1914.

[24] Labarta E, Sebastian-Leon P, Devesa-Peiro A, Celada P, Vidal $\mathrm{C}$, Giles J, et al. Analysis of serum and endometrial progesterone in determining endometrial receptivity. Human Reproduction. 2021; 36: 2861-2870.

[25] Bourgain C, Devroey P, Van Waesberghe L, Smitz J, Van Steirteghem AC. Effects of natural progesterone on the morphology of the endometrium in patients with primary ovarian failure. Human Reproduction. 1990; 5: 537-543.

[26] De Ziegler D, Bulletti C, De Monstier B, Jääskeläinen AS. The first uterine pass effect. Annals of the New York Academy of Sciences. 1997; 828: 291-299.

[27] Cicinelli E, de Ziegler D, Bulletti C, Matteo MG, Schonauer LM, Galantino P. Direct transport of progesterone from vagina to uterus. Obstetrics and Gynecology. 2000; 95: 403-406.

[28] Labarta E, Mariani G, Rodríguez-Varela C, Bosch E. Individualized luteal phase support normalizes live birth rate in women with low progesterone levels on the day of embryo transfer in artificial endometrial preparation cycles. Fertility and Sterility. 2021. (in press)

[29] Cédrin-Durnerin I, Isnard T, Mahdjoub S, Sonigo C, Seroka A, Comtet M, et al. Serum progesterone concentration and live birth rate in frozen-thawed embryo transfers with hormonally prepared endometrium. Reproductive BioMedicine Online. 2019; 38: $472-480$. 